\title{
Summary of the main results of the KASCADE and KASCADE-Grande exper- iments
} \author{
J. Zabierowski ${ }^{12}$ \\ ${ }^{1}$ Dipartimento di Fisica, Università degli Studi di Torino, Italy \\ ${ }^{2}$ Institut für Kernphysik, KIT - Karlsruhe Institute of Technology, Germany \\ ${ }^{3}$ Universidad Michoacana, Inst. Física y Matemáticas, Morelia, Mexico \\ ${ }^{4}$ Institut für Experimentelle Kernphysik, KIT - Karlsruhe Institute of Technology, Germany \\ ${ }^{5}$ Horia Hulubei National Institute of Physics and Nuclear Engineering, Bucharest, Romania \\ ${ }^{6}$ Osservatorio Astrofisico di Torino, INAF Torino, Italy \\ ${ }^{7}$ Universidade São Paulo, Instituto de Física de São Carlos, Brasil \\ ${ }^{8}$ Fachbereich Physik, Universität Wuppertal, Germany \\ ${ }^{9}$ Department of Physics, Siegen University, Germany \\ ${ }^{10}$ Dept. of Astrophysics, Radboud University Nijmegen, The Netherlands \\ ${ }^{11}$ National Centre for Nuclear Research, Department of Astrophysics, Lodz, Poland \\ ${ }^{12}$ Frankfurt Institute for Advanced Studies (FIAS), Frankfurt am Main, Germany \\ ${ }^{13}$ Department of Physics, University of Bucharest, Bucharest, Romania
}

A. Chiavassa ${ }^{1 *}$, W.D. Apel ${ }^{2}$, J.C. Arteaga-Velázquez ${ }^{3}$, K. Bekk ${ }^{2}$, M. Bertaina ${ }^{1}$, J. Blümer ${ }^{2,4 * *}$, H. Bozdog ${ }^{2}$, I.M. Brancus ${ }^{5 * *}$, E. Cantoni ${ }^{1,6}$, F. Cossavella ${ }^{4}$, K. Daumiller ${ }^{2}$, V. de Souza ${ }^{7}$, F. Di Pierro ${ }^{1}$, P. Doll ${ }^{2}$, R. Engel ${ }^{2}$, D. Fuhrmann ${ }^{8}$, A. Gherghel-Lascu ${ }^{5}$, H.J. Gils ${ }^{2}$, R. Glasstetter ${ }^{8}$, C. Grupen ${ }^{9}$, A. Haungs ${ }^{2}$, D. Heck ${ }^{2}$, J.R. Hörandel ${ }^{10}$, D. Huber ${ }^{4}$, T. Huege ${ }^{2}$, K.-H. Kampert ${ }^{8}$, D. Kang ${ }^{2}$, H.O. Klages ${ }^{2}$, K. Link ${ }^{4}$, P. Łuczak ${ }^{11}$, H.J. Mathes ${ }^{2}$, H.J. Mayer ${ }^{2}$, J. Milke ${ }^{2}$, B. Mitrica ${ }^{5}$, C. Morello ${ }^{6}$, J. Oehlschläger ${ }^{2}$, S. Ostapchenko ${ }^{12}$, N. Palmieri ${ }^{4}$, T. Pierog ${ }^{2}$, H. Rebel $^{2}$, M. Roth $^{2}$, H. Schieler ${ }^{2}$, S. Schoo ${ }^{2}$, F.G. Schröder ${ }^{2}$, O. Sima ${ }^{13}$, G. Toma ${ }^{5}$, G.C. Trinchero ${ }^{6}$, H. Ulrich ${ }^{2}$, A. Weindl ${ }^{2}$, J. Wochele ${ }^{2}$, and

\begin{abstract}
The KASCADE and KASCADE-Grande experiments operated in KIT-Campus North, Karlsruhe (Germany) from 1993 to 2012. The two experiments studied primary cosmic rays in the energy range from $10^{14}$ $\mathrm{eV}$ to $10^{18} \mathrm{eV}$, investigating the change of slope of the spectrum detected at $2-4 \times 10^{15} \mathrm{eV}$, the so called knee. We briefly review the performance of the experiments and then the main results obtained in the operation of both experiments: the test of hadronic interaction models, the all particle primary spectrum, the elemental composition of primary cosmic rays (with the first claim of a knee-like feature of the heavy primaries spectrum) and the search for large scale anisotropies.
\end{abstract}

\section{Introduction}

In this contribution we will briefly review the major results of the KASCADE [1] and KASCADE-Grande [2] experiments. The two experiments were located at the KIT-Campus North, Karlsruhe (Germany) $49.1^{\circ} \mathrm{N}, 8.4^{\circ} \mathrm{E}$, $110 \mathrm{~m}$ a.s.l., the data taking started in 1993 (only KASCADE), then was extended to KASCADE-Grande in 2003 and ended in late 2012.

The project started with the KASCADE experiment to study primary cosmic rays in the $10^{14}-10^{16} \mathrm{eV}$ energy range, then was extended to KASCADE-Grande to cover the $10^{16}-10^{18} \mathrm{eV}$ interval. Both arrays were high resolution multi component Extensive Air Shower (EAS) detectors. This feature allowed to perform data analysis intro-

\footnotetext{
*e-mail: andrea.chiavassa@to.infn.it

** Now: Head of KIT Division V - Physics and Mathematics

$* * *$ deceased
}

ducing, in the high energy cosmic ray field, the passage from the use of the moments of the EAS parameters distributions to the use of the shapes of these distributions.

In parallel to the construction of the experiments at the KIT laboratories the CORSIKA [3] EAS simulation code was developed; the performance of this code including different high and low energy hadronic interaction models also played a very important role in the development of the data analysis. CORSIKA is nowadays recognized by the international community as the reference code for EAS simulations.

The KASCADE complex was also devoted to the test and development of new EAS detection techniques, among the others we want to mention: LOPES [4] a radio antenna field measuring signals generated in the EAS development in the $40-80 \mathrm{MHz}$ band and the microwave experiment CROME [5]. 
The data of the two experiments are now stored, and are available to the public via a customized web page, in the KCDC (KASCADE Cosmic-ray Data Center) [6].

In this paper we will briefly review the main characteristics of both experiments and then we will summarize the main results achieved.

In this conference proceedings the latest KASCADEGrande results can be found in the contribution by Kang et al. [7] and by Arteaga et al. [8].

\section{The experiments}

\subsection{KASCADE}

A schematic layout of the KASCADE experiment is shown in figure 1 . The three main components can be briefly summarized in:

- A scintillator array comprising 252 detector stations of electron and muon counters arranged on a grid of $200 \times$ $200 \mathrm{~m}^{2}$ and providing in total about $500 \mathrm{~m}^{2}$ of $e-\gamma$ and $620 \mathrm{~m}^{2}$ of $\mu$ detector coverage. The detection thresholds for vertical incidence are $E_{e}>5 \mathrm{MeV}$ and $E_{\mu}>230$ $\mathrm{MeV}$.

- A central detector system $\left(320 \mathrm{~m}^{2}\right)$ consisting of a highly-segmented hadronic calorimeter read out by 44,000 channels of warm liquid ionization chambers distributed over 9 read-out layers, another set of scintillation counters above the shielding (top cluster), a trigger plane of scintillation counters in the third iron gap and, at the very bottom, 2 layers of positional sensitive MWPC's, and a streamer tube layer with pad read-out for investigation of the muon component at $E_{\mu}>2.4$ $\mathrm{GeV}$.

- A $48 \times 5.4 \mathrm{~m}^{2}$ tunnel housing three horizontal and two vertical layers of positional sensitive limited streamer $E_{\mu}>0.8 \mathrm{GeV}$.

A complete description of the experiment and of its performance can be found in [1]. Here we briefly remember that the array determines, for each event: the shower geometry (core position and arrival direction), the shower size ( $N_{e}$, total number of electrons at observation level) and the truncated muon number $\left(N_{\mu}^{t r}\right.$, muon number between 40 and $200 \mathrm{~m}$ from the shower core).

The KASCADE reconstruction accuracy has been calculated analyzing simulated events. The precision achieved are: core position $\sigma_{r}<5 \mathrm{~m}$, arrival direction $\sigma_{\Phi}<0.5^{\circ}$, shower size $\sigma_{N_{e}}<5 \%$ for $L g N_{e}>4.5$ (see figure 2), truncated muon number $\sigma_{N_{\mu}^{t r}}<5 \%$ for $L g N_{\mu}^{t r}>4$. The total energy deposited by hadrons in the central calorimeter was sampled with an error lower than $15 \%$.

\subsection{KASCADE-Grande}

The KASCADE experiment was then extended installing, on a $0.5 \mathrm{~km}^{2}$ area, over an irregular triangular grid with an average spacing of $137 \mathrm{~m}$, an array, named Grande: 37 plastic detector stations of $10 \mathrm{~m}^{2}$ active area each. The experiment layout is shown in figure 3 .

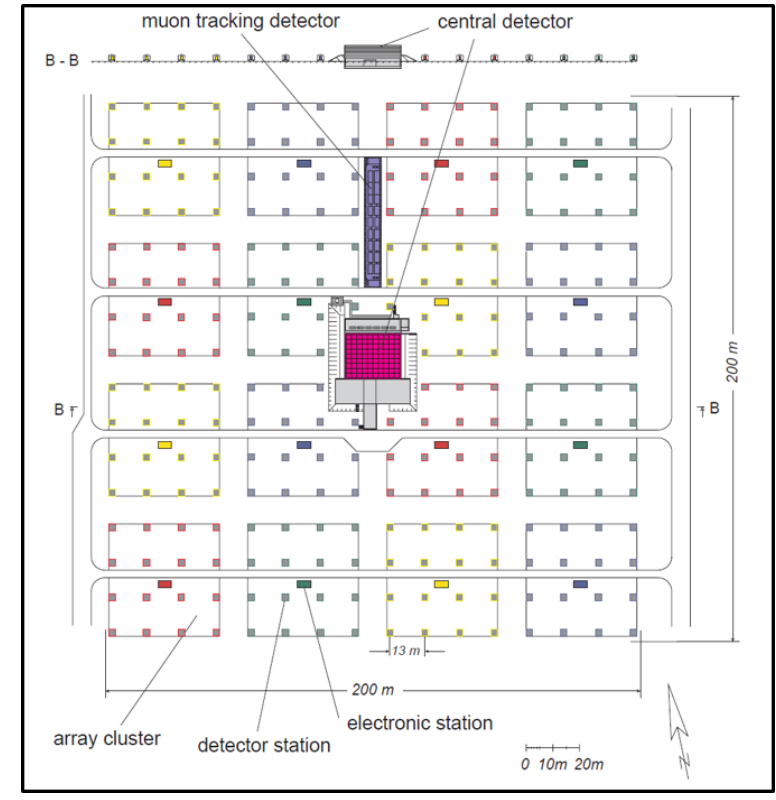

Figure 1. Schematic layout of the KASCADE experiment.

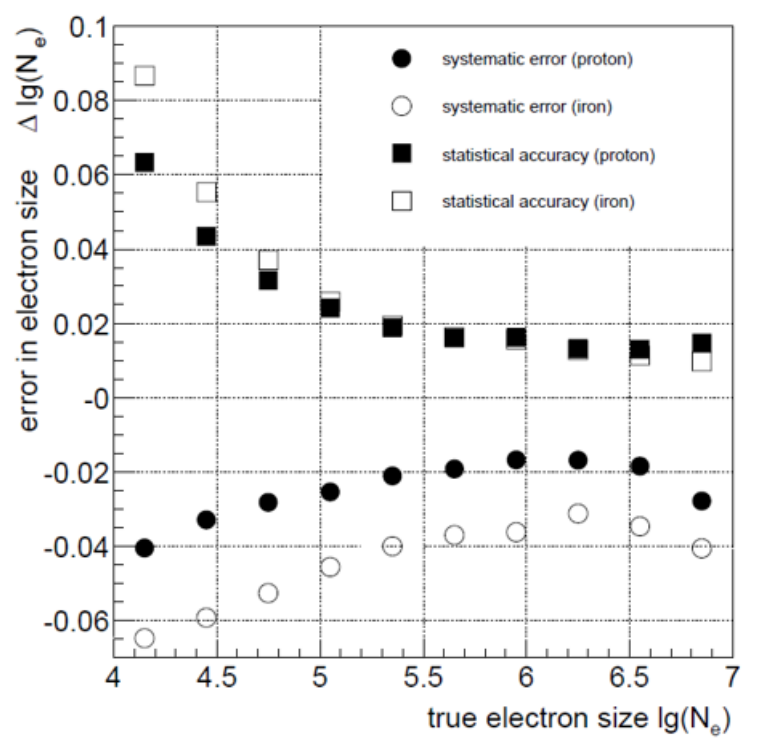

Figure 2. Average difference (systematic error) and rms-width (statistical accuracy) of reconstructed $L g N_{e}$ versus the particle number for simulated showers. Filled symbols refer to results obtained from showers simulated for $\mathrm{H}$ primary particles, while open symbols to Fe primaries.

Grande provided the measurement of the shower geometry and of the total number of charged particles $\left(N_{c h}\right)$, the number of muons $\left(N_{\mu}\right)$ was sampled by the previously discussed KASCADE $\mu$ detectors.

The full description of the experiment and of the event reconstruction can be found in [2]. The Grande array precision has been studied comparing the reconstruction of the EAS parameters independently obtained, for real events, by the KASCADE and Grande arrays. The obtained accuracies are: $<10 \mathrm{~m}$ on the core position, $\sim 1^{\circ}$ on the arrival direction and $\sim 10 \%$, above the $100 \%$ detec- 


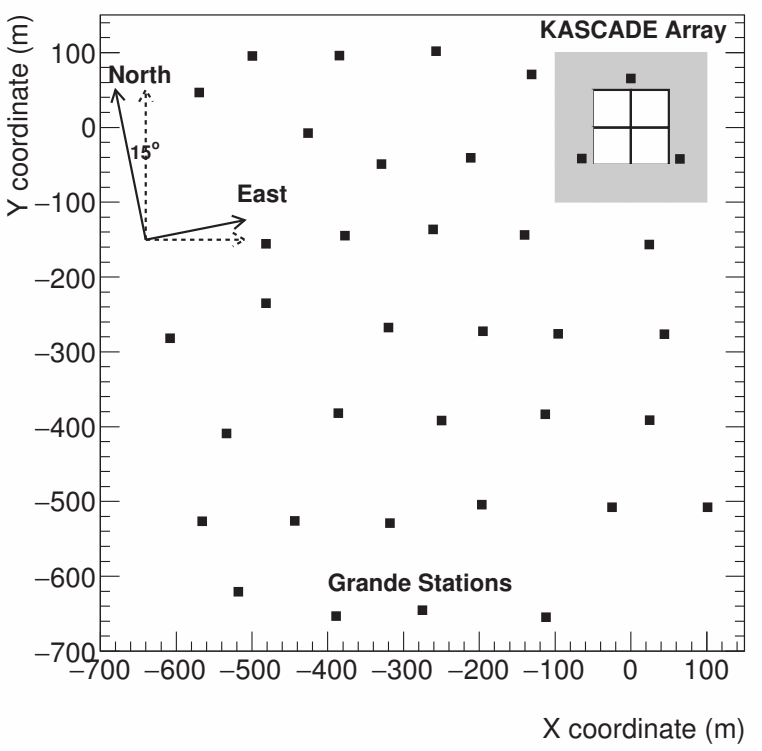

Figure 3. Layout of the KASCADE-Grande experiment.

tion efficiency, on $N_{c h}$ (with a systematic difference from KASCADE lower than $5 \%$ ). The muon number $N_{\mu}$ was determined with a $<20 \%$ error.

\section{Main Results}

\subsection{Test of Hadronic Interaction Models}

Since the beginning of the KASCADE operation great attention was devoted to a careful check of the hadronic interaction models used in the CORSIKA simulation. This was pursued studying the correlations between the main observables of the central detector (number of hadrons, hadronic energy sum) and of the KASCADE array (shower size, truncated muon number) $[9,10]$. We can summarize the results saying that no model gave a complete description of the data. These results were then used to tune the hadronic interaction models (we must keep in mind that all these analyses were performed before the beginning of the LHC operation).

\subsection{All particle spectrum}

A composition independent all-particle energy spectrum of cosmic rays was reconstructed in the energy range of $10^{16} \mathrm{eV}$ to $10^{18} \mathrm{eV}$ from the Grande data within a total uncertainty in flux of $10-15 \%$ [11]. The spectrum is, in the overlapping region, in agreement with the earlier published spectrum by KASCADE [12].

Significant structures are observed in the spectrum, beside the well known one at a few times $10^{15} \mathrm{eV}$, i.e. the so called "knee", there is also clear evidence that just above $10^{16} \mathrm{eV}$ the spectrum shows a significant 'concave' behavior. Another feature in the spectrum is a small break, i.e. knee-like feature, at around $10^{17} \mathrm{eV}$. The latter slope

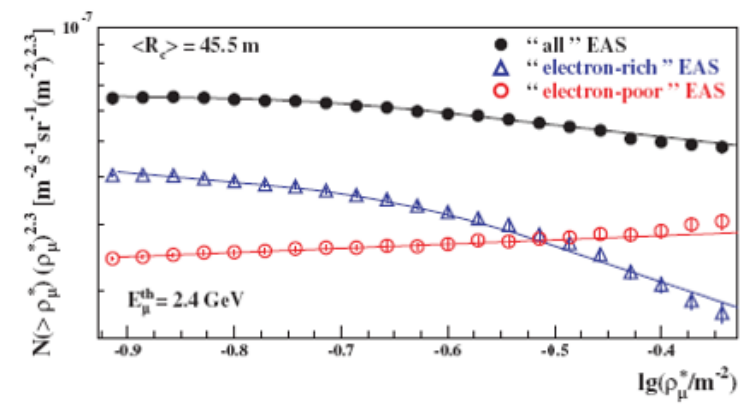

Figure 4. Spectra measured, in terms of the muon density at $\sim 45 \mathrm{~m}$ from the shower core, for the electron-rich and electronpoor event sample. The all particle spectrum is also shown as reference.

change occurs at an energy where the rigidity dependent, i.e. charge dependent, knee of the iron component would be expected, if the 'knee' is caused by light primaries.

\subsection{Elemental composition of cosmic rays}

Already in 2005 KASCADE could prove [12] that the knee is caused by a decrease of the light mass group of primary particles and not by medium and heavy primary particles. This result has been obtained unfolding the spectra of five different primaries $(\mathrm{H}, \mathrm{He}, \mathrm{C}, \mathrm{Si}, \mathrm{Fe})$ from the measured bi-dimensional spectrum $\left(L g N_{e}\right.$ vs $\left.L g N_{\mu}^{t r}\right)$. It was shown that the details of this result depend on the hadronic interaction model used in the EAS simulation $[12,13]$, but that the general trend of spectra showing the change of slope at an energy increasing with the charge (mass) of the primary particle holds independently from the hadronic interaction model.

An alternative analysis, separating events in the "electron rich" (i.e. light primaries) and "electron poor" (i.e. heavy primaries) samples, was introduced by the KASCADE collaboration [14]. The event separation was based on the ratio between the muon and electron numbers converted, using the constant intensity cut technique, at a reference atmospheric depth (i.e zenith angle). Even if this technique is less powerful than the previously discussed unfolding, its results have a much smaller dependence on the hadronic interaction model used in the EAS simulation. Separating the events according to the value of this ratio, the spectrum of the two event sample can be measured. In figure 4 the spectrum is shown in terms of the measured muon density $\left(\rho_{\mu}^{*}\right)$ at fixed core distance $r \sim 45$ $\mathrm{m}$. The muon density is obtained dividing the number of tracked muons $\left(E_{\mu}>2.4 \mathrm{GeV}\right)$ by the total sensitive area. Only the spectrum of the "electron rich" sample shows the change of slope: confirming that the knee of the primary spectrum has to be ascribed to the light primary component.

With KASCADE-Grande we investigated such individual mass group spectra also at higher primary energies $[15,16]$. All the simulations for the described analyses are performed with the CORSIKA EAS simulation code with various hadronic interaction models. The application 


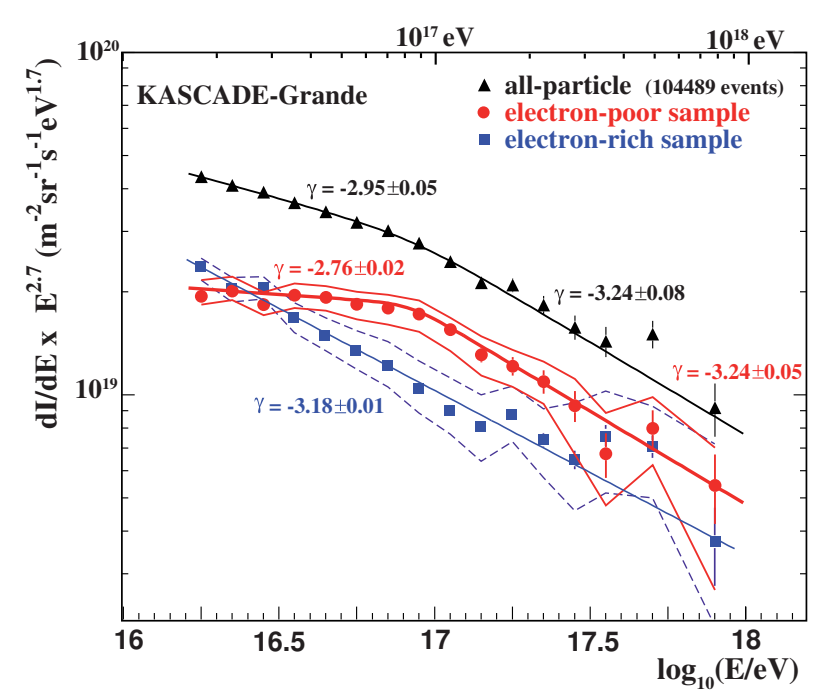

Figure 5. Reconstructed energy spectrum of the electron-poor and electron-rich components together with the all-particle spectrum for the angular range $0^{\circ}-40^{\circ}$. The error bars show the statistical uncertainties; the bands assign systematic uncertainties due to the selection of the subsamples. Fits on the spectra and resulting slopes are also indicated.

of this methodical approach to shower selection and separation in various mass groups were performed and crosschecked in different ways. The reconstructed spectrum of the electron-poor events, i.e. the spectrum of heavy primaries, shows a distinct knee-like feature at about $8 \times 10^{16}$ $\mathrm{eV}$ with a statistical significance of $3.5 \sigma$ [15] (see figure 5). The analysis was repeated on the basis of different hadronic interaction models [17]. Despite the fact, that the relative abundance of the heavy particles varies significantly dependent on the model in use, the spectral feature of this "heavy" knee is visible in all the spectra. This fits to a rapidity dependent knee scenario with the results of KASCADE, where a reduction of the light component beyond the first knee was observed. In addition, an ankle-like feature was observed in the spectrum of the electron-rich events, i.e. light elements of the primary cosmic rays, at an energy of $10^{17.08 \pm 0.08} \mathrm{eV}$ [16] (see figure 6). At this energy, the spectral index changes by $\Delta \gamma=0.46$ with a statistical significance of $5.8 \sigma$.

\subsection{Large scale anisotropies}

A search for large scale anisotropies has been performed, in different energy ranges, with both experiments. None of them gave positive results, therefore upper limits were derived. Those obtained by the former array KASCADE in the $700-6000 \mathrm{TeV}$ energy range were published in 2004 [18] and were afterward overtaken by the positive detection, in the same energy range, of a large scale anisotropy by larger experiments, like Ice-Top [19].

The KASCADE-Grande data were analyzed following the East-West method [20] searching for a dipole large scale anisotropy in the $10^{15}-10^{17} \mathrm{eV}$ energy range. The event statistics accumulated during the whole KASCADEGrande data taking was not sufficient to reach a positive

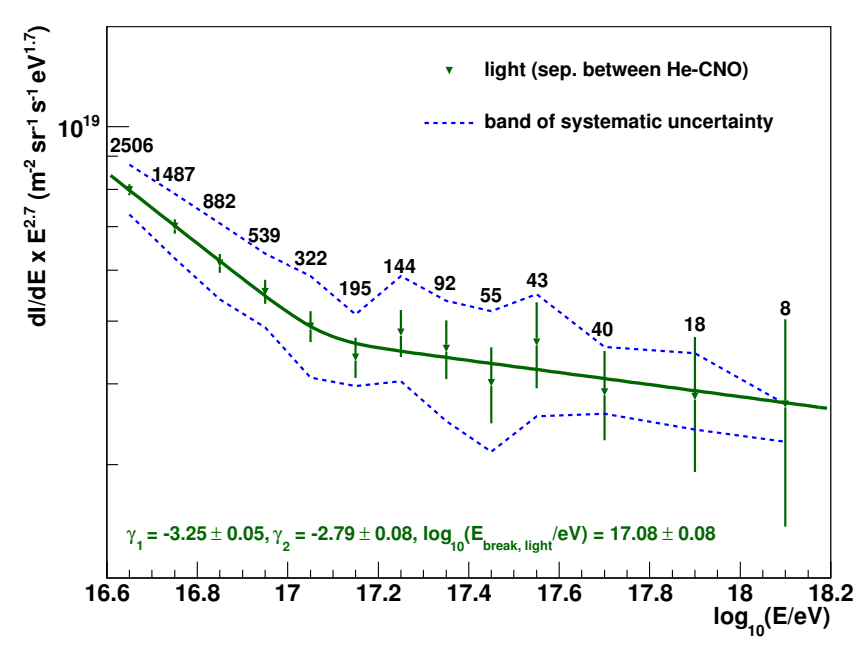

Figure 6. The reconstructed energy spectrum of the light mass component of cosmic rays. The number of events per energy bin is indicated as well as the range of systematic uncertainty. The error bars show the statistical uncertainties.

Table 1. First harmonic amplitude and phase in different intervals of $N_{c h}$ (number of charged particles at observation level).

\begin{tabular}{llll}
\hline$L g\left(N_{c h}\right)$ & $\begin{array}{l}\text { Median } \\
\text { Energy }(\mathrm{eV})\end{array}$ & $A \times 10^{-2}$ & $\begin{array}{l}\text { Phase } \\
(\mathrm{deg})\end{array}$ \\
$5.2-5.6$ & $2.7 \times 10^{15}$ & $0.26 \pm 0.10$ & $225 \pm 22$ \\
$5.6-6.4$ & $6.1 \times 10^{15}$ & $0.29 \pm 0.16$ & $227 \pm 30$ \\
$\geq 6.4$ & $3.3 \times 10^{16}$ & $1.2 \pm 0.9$ & $254 \pm 42$
\end{tabular}

detection, and also in this case we could only derive upper limits [21]. Up to now these results are the only ones available in this energy range and the phase of the dipole derived, with large errors, in different energy intervals confirm the phase flip observed at energies above $10^{14} \mathrm{eV}$ by various experiments[19, 22, 23]. The KASCADE-Grande results are reported in table 1.

\section{Conclusions}

In this report we have summarized the main results obtained by the KASCADE and KASCADE-Grande experiments. A great relevance in the development of the analysis techniques that brought to all these results is due to the CORSIKA EAS simulation. In this sense the first results were concentrated on the test of the hadronic interaction models used in CORSIKA.

The KASCADE and KASCADE-Grande results indicate that the knee of the primary spectrum is due to light primaries and that heavier elements show a similar spectral feature at higher energy (scaling approximately with the charge of the primary particle). These results favor the models explaining the knee as the end of the containment of primary particles inside magnetic fields.

\section{References}

[1] T. Antoni et al·, Nucl. Instr. and Meth. A 513, 490 (2003). 
[2] W.D. Apel et al., Nucl. Instr. and Meth. A 620, 202 (2010).

[3] D. Heck et al., Report FZKA 6019, Forschungszentrum Karlsruhe (1998).

[4] H. Falke et al., Nature 435, 313 (2005).

[5] R. Smida et al., PRL 113, 221101 (2014).

[6] A. Haungs et al., Eur. Phys. J. C, 78, 741 (2018).

[7] D. Kang et al., this Proceedings.

[8] J.C. Arteaga et al., this Proceedings.

[9] W.D. Apel et al., J. Phys.G: Nucl. Part. Phys 34, 2581 (2007).

[10] W.D. Apel et al., J. Phys.G: Nucl. Part. Phys 36, 035201 (2009).

[11] W.-D. Apel et al., Astropart. Phys. 36, 183 (2012).

[12] T. Antoni et al., Astropart. Phys. 24, 1 (2005).
[13] W.D. Apel et al., Astropart. Phys. 31, 86 (2009).

[14] T. Antoni et al., Astropart. Phys. 16, 373 (2002).

[15] W.D. Apel et al., Phys. Rev. Lett. 107, 171104 (2011).

[16] W.D. Apel et al., Phys. Rev. D, 87, 081101(R) (2013).

[17] W.D. Apel et al. - KASCADE-Grande, Adv. Space Res. 53, 1456 (2014)

[18] T. Antoni et al., ApJ 604, 687 (2004).

[19] M.G. Aartsen et al., ApJ 765, 55 (2013).

[20] R. Bonino et al., ApJ. 738, 67 (2011).

[21] A. Chiavassa et al., J. Phys. Conf. Series 531, 012001 (2014).

[22] M. Aglietta et al., ApJ 692, L130 (2009).

[23] M. Amenomori et al., ApJ 836, 153 (2017). 